\title{
Improvement in line orientation discrimination is retinally local but dependent on cognitive set
}

\author{
LING-PO SHIU and HAROLD PASHLER \\ University of California, San Diego, La Jolla, California
}

\begin{abstract}
The ability of human observers to discriminate the orientation of a pair of straight lines differing by $3^{\circ}$ improved with practice. The improvement did not transfer across hemifield or across quadrants within the same hemifield. The practice effect occurred whether or not observers were given feedback. However, orientation discrimination did not improve when observers attended to brightness rather than orientation of the lines. This suggests that cognitive set affects tuning in retinally local orientation channels (perhaps by guiding some form of unsupervised learning mechanism) and that retinotopic feature extraction may not be wholly preattentive.
\end{abstract}

The analysis of simple features in a scene is commonly believed to occur at an early stage of visual perception. Several different experimental techniques, such as Treisman's visual search paradigm (e.g., Treisman \& Gelade, 1980), have shown that detection of a distinctive visual feature (e.g., a vertical line among a background of horizontal lines) is fast and easy and that it does not appear to depend on serial attentional processes. Experiments on transfer of adaptation effects (Blakemore \& Campbell, 1969 ) and on redundancy effects in classification (Garner, 1974) suggest that the underlying mechanisms respond selectively to different target features (such as color and orientation) (see review by Treisman, 1986). It is tempting to assume that all these experimental results reflect neural mechanisms that respond to specific visual features. Neurons selectively tuned for features such as contour orientation, direction of motion, and color have been identified in cortical visual areas including V1, V2, and V4 in monkeys (Van Essen \& Maunsell, 1983). The general picture that has emerged from these studies is that of an early, featural analysis stage that operates in parallel across the visual field, independently of attention.

A few investigators have examined the changes in the efficiency of visual feature analysis that occur with practice. With training, human observers have shown substantial and persistent improvement in making fine discriminations of various simple visual features, including direction of motion, spatial frequency, and orientation of texture boundaries (see, e.g., Ball \& Sekuler, 1987;

We thank Norman Anderson, Mark Carrier, MaryLou Cheal, Jeff Miller, Dov Sagi, and an anonymous reviewer for helpful comments on an earlier draft of this paper, and Alann Lopes for technical assistance. Parts of the results were presented at the meeting of the Association for Research in Vision and Ophthalmology, in Sarasota, FL (April 1991). This research was supported by the Office of Naval Research under contracts NO0014-88-K-0281 and N00014-91-J-1735. Correspondence should be addressed to H. Pashler, Department of Psychology, 0109, University of California, San Diego, La Jolla, CA 92093 (e-mail: hpashler@ucsd.edu [internet], hpashler@ucsd [bitnet]).
Fiorentini \& Berardi, 1981; Karni \& Sagi, 1991). The improvement is often specific to various stimulus parameters. For example, the training effect given to one eye may not be transferred to the other eye (Karni \& Sagi, 1991). This kind of parameter-specific perceptual learning has implications not only for the theory of feature analysis but also for the understanding of the neural mechanisms mediating the processes. For example, a practice effect specific to the retinal location being trained would seem to imply the involvement of some neural mechanisms that are retinotopically organized.

In this study, we investigated whether the sensitivity of human observers to contour orientation can be improved with training. Contour orientation seems to be one of the most important components in early mammalian vision (Hubel \& Wiesel, 1962). It might be advantageous if the ability to discriminate orientation were modifiable with perceptual experiences. Blakemore and Cooper (1970) have demonstrated that kittens exposed only to contours of one orientation during a critical period of development are greatly impaired in seeing contours of other orientations. This behavioral impairment is accompanied by permanent changes in the responses of orientationselective neurons in the cat's visual cortex. While these studies reported sensitivity loss as a result of seriously deprived and disrupted visual experience, we attempted to increase sensitivity by enriching the perceptual input that the adult human observer receives.

The orientation columns in primates' striate cortex are topographically organized. If improvement with orientation discrimination is localized to the trained retinal region, it would suggest the involvement of these or other similarly organized neural mechanisms. We also looked at the conditions required for perceptual learning. If orientation extraction takes place entirely within a preattentive stage of vision, as suggested by Treisman and Gelade (1980), then learning effects may also be independent of any form of attentional or cognitive control. On the other hand, more recent evidence (see, e.g., Treisman \& Gormican, 1988) suggests that discrimination of small orien- 
tation differences requires serial attention. If the latter view is correct, then learning about orientation differences might also be dependent on some kind of cognitive control.

\section{GENERAL METHOD}

\begin{abstract}
We set out to train some human observers to discriminate a small orientation difference between a pair of straight lines. The lines were inclined at either $9.8^{\circ}$ or $7^{\circ}$ counterclockwise from the vertical axis. They were approximately $1.5 \mathrm{~cm}$ long, subtending a visual angle of $1.42^{\circ}$ at a viewing distance of $60 \mathrm{~cm}$. Each line was presented at either the top-left or the bottom-right corner of a cathoderay tube (NEC MultiSync 2A) controlled by an IBM PC. The width of the lines equaled 1 pixel. The distance from the midpoint of a line to the fixation cross was $8.4 \mathrm{~cm}$, or $7.94^{\circ}$. The midpoint of a line and the fixation point formed a rectangle of approximately $5 \times 7 \mathrm{~cm}^{2}$. The luminance of the lines was $50 \mathrm{~cd} / \mathrm{m}^{2}$, against a background luminance of $1 \mathrm{~cd} / \mathrm{m}^{2}$.
\end{abstract}

Observers were given either a matching task or an identification task. In the matching task, two lines were presented successively for $45 \mathrm{msec}$ each, separated by a 300 -msec blank interval. Observers reported after each trial whether the lines were the same or different in orientation. In the identification task, only one of the two lines was presented in a trial. Observers identified which one it was by pressing one of two buttons.

In all experiments, observers maintained fixation at the center of the display. Eye movement was further controlled by requiring them to report an alphanumeric character (either $S$ or 5), which replaced the fixation cross on some catch trials. The alphanumeric character had a height of $0.8 \mathrm{~cm}$, and it subtended a visual angle of $.76^{\circ}$. The exposure time was $45 \mathrm{msec}$. Accuracy in this recognition task was emphasized. In order to make the subjects feel comfortable, no chinrest or bite bar was employed.

Each session of training consisted of 12 blocks of 44 trials (including 4 catch trials). The two oriented lines appeared equally often within a block. The matching task consisted of the same number of same and different trials. Response accuracy was emphasized. The subjects were allowed as much time as they needed for making a response. The next trial started $1.5 \mathrm{sec}$ after a response was made. They were given a rest period of at least $40 \mathrm{sec}$ at the end of each block. They were encouraged to rest until they felt comfortable enough to continue.

All subjects were undergraduates at the University of California, San Diego. They participated either in return for payment, or in partial fulfillment of a course requirement.

\section{EXPERIMENT 1}

In an experiment concerning the oblique effect in line orientation judgments, Vogels and Orban (1985) found that the just noticeable difference in orientation discrimination improves with practice for oblique orientations. In the present experiment, we asked whether the practice effect would be specific to the retinal location. Previous investigations have yielded practice effects that were retinally specific for tasks requiring judgment of direction of motion (Ball \& Sekuler, 1987), spatial frequency (Fiorentini \& Berardi, 1981), and orientation of texture boundaries (Karni \& Sagi, 1991).

Our subjects were trained in different feedback conditions. The importance of feedback in perceptual learning is not clear. McKee and Westheimer (1978) obtained a practice effect in vernier acuity, whether or not they gave their subjects feedback. On the other hand, Ball and Sekuler (1987) found that subjects deprived of trial-bytrial feedback during training did not improve in their accuracy of discriminating the directions of some moving dots (for oblique orientations).

\section{Method}

The subjects were given either the matching task or the identification task. They were divided into three groups, according to the level of feedback they received. One group of observers received no feedback at all; another group got feedback only at the end of each block (percentage of correct responses); and the final group had trial-by-trial feedback (a warning tone after every incorrect response) plus feedback at the end of each block. Accuracy of the response was emphasized.

To determine whether the learning can be transferred to an untrained retinal location at equal eccentricity, all observers were trained with the lines at one corner (for nine blocks) and tested at another corner (for three blocks).

\section{Results and Discussion}

Fifty-nine and 36 observers participated in the matching and identification tasks, respectively. Those who made more than 5 errors (out of 48 ) in recognizing the central alphanumeric characters were excluded from analysis (11 in the matching and 6 in the identification task; of these, 3 were in trial feedback, 6 in block feedback, and 8 in no feedback). The subjects included in the analyses made 1.5 errors (or $3.1 \%$ ) on the average.

The results are shown in Figures $1 \mathrm{~A}$ and 1B. The two graphs are quite similar and statistical analyses yielded similar results, so only the statistics for the matching task are reported here. Observers given trial and block feedback showed $10.2 \%$ and $7.8 \%$ increase in accuracy at the end of practice, both of which were statistically significant $[F(1,15)=13.39, p<.01 ; F(1,15)=6.04$, $p<.05]{ }^{1}$

When the lines appeared at a new location, accuracy dropped significantly $[F(1,15)=4.58$, and $F(1,15)=$ $6.25, p<.05]$ and was not much different from that when training started $(<2 \%){ }^{2}$ It is quite clear that these practice effects did not transfer to a new location (at equal eccentricity) in the opposite hemifield. A further experiment indicated no transfer across quadrants within the same hemifield either. Sixteen new subjects trained with trial feedback showed a significant drop of $8.8 \%$ in accuracy $[F(1,15)=7.34, p<.05]$ when the lines shifted to a new quadrant in the same hemifield. Accuracies at the beginning of training and testing were $62 \%$ and $60 \%$, respectively.

Figure 1A also shows that observers who were given no feedback during training did not improve. The difference between the beginning and the end of training is only $0.7 \%$, which is not significant $[F(1,15)<1]$. However, when the lines appeared in a new location, discrimination accuracy dropped by $4 \%$. This decrement in the transfer phase is more evident in the identification task (Figure 1B).

As described above, eye movement was controlled by having subjects report a central alphanumeric character 


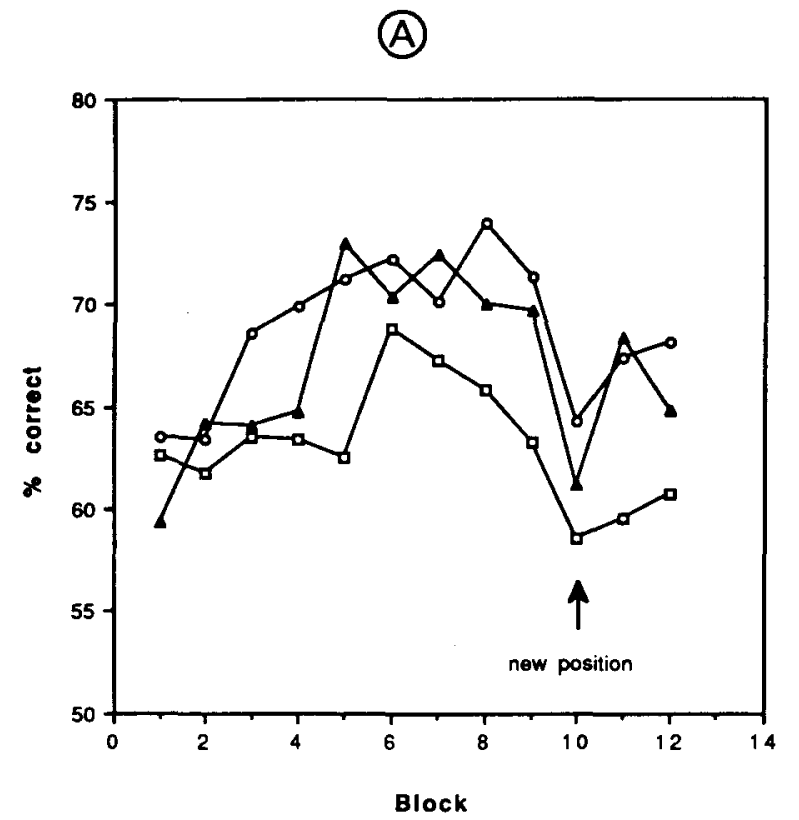

(B)

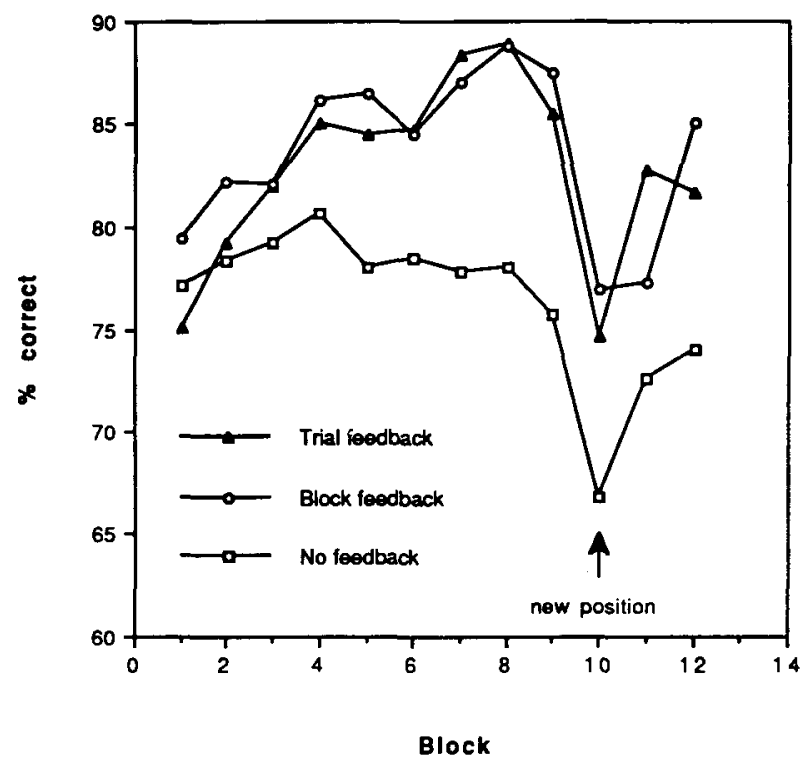

Figure 1. Percentages of correct response in (A) the matching task and (B) the identification task in Experiment 1. Each data point represents the mean percentage correct for a block of $\mathbf{4 0}$ trials. Data for each feedback condition were pooled over 16 observers in the matching task and 10 observers in the identification task. The lines appeared at a new location after Block 10.

(S or 5) in some catch trials. The data from subjects who made more than 5 errors were discarded from analysis. It is unlikely that the subjects improved in orientation discrimination accuracy by moving their eyes closer to the lines, so that the lines fell on a part of the retina with higher acuity. If they had done that, they should have shown a large transfer effect when the lines appeared in another corner, if they moved their eyes toward the new location too. Nevertheless, we did a control experiment to show that the central alphanumeric character task provided a good control of eye movement.

A new group of 6 subjects was given the same matching task. But this time, the subjects were told to fixate at the location of the lines. They were also asked to report the $S$ or 5 in some random catch trials. This group of subjects failed on the average to recognize $41.3 \%$ of the characters. Although this is significantly better than the $50 \%$ chance level [one-sample $t(5)=2.34, p<.05$ ], it is far worse than our criterion (10.4\%). For this group of subjects, the accuracy in orientation discrimination improved by $21 \%$ after training $(56.2 \%$ and $77 \%$ at the beginning and the end of training). As expected, their performance did not decrease when the lines moved to a new location $[F(1,5)<1]$. In fact, it was slightly higher at the beginning of transfer $(78.5 \%)$. In short, this group of subjects, who were allowed to look at the lines directly, showed a pattern of results that was very different from that in our original experiment. Therefore, we conclude that eye movement is not likely to influence the interpretation of our results.

\section{EXPERIMENT 2}

Performance in the no-feedback condition appears puzzling at first glance. On the one hand, there was no obvious sign of learning: accuracy in the trained location did not improve. On the other hand, the position change did impair accuracy, as in the other conditions. The most plausible explanation is simply that fatigue or some other nonspecific decrement was superimposed on the (positionspecific) learning effect (Kimble \& Shatel, 1952; Woodworth \& Schlosberg, 1955). This would also account for the improvement when block feedback was given, which sufficed to maintain motivation without the observers' being informed of the correctness of their judgments about any individual stimulus.

If this is correct, performance without feedback should improve over multiple sessions, since fatigue effects would dissipate between sessions. Karni and Sagi (1991), investigating discrimination of the orientation of texture boundaries, also trained their subjects without feedback. They noted that there was no increment in performance within sessions, but they found improvement from one session to the next.

\section{Method}

Three observers (M.L., A.S., and P.P.) were given multiple sessions of training without feedback. Subjects performed the matching task. A.S. and M.L. were trained with the lines at the top-left and bottom-right corners, respectively. The lines were the same as those used in the last experiment. P.P. was given lines that extended horizontally and inclined at either $11.5^{\circ}$ or $7.7^{\circ}$ counterclockwise from the horizontal axis. The lines appeared at the top-left corner throughout training. A.S. and P.P. were given two additional testing days. On one of the 2 days of testing, the lines were shown in an untrained corner in the even-numbered blocks. On the 
even-numbered blocks of the other testing day, the orientation of the lines was changed by $90^{\circ}$ (i.e., the lines with which A.S. was trained were given to P.P., and vice versa). On both days, the lines appeared at the trained location and orientation in the odd-numbered blocks. To ensure that observers were fixating on the cross, a different pair of alphanumeric characters (S or $5, \mathrm{~B}$ or 8 , I or $1, \mathrm{G}$ or 6 , and $\mathrm{Z}$ or 2 ) was used on each day. ${ }^{3}$

\section{Results and Discussion}

The three observers (M.L., A.S., and P.P.) were very accurate with the alphanumeric character task. M.L., who was the least accurate, made only two errors per day. Fig- ure 2 shows their results from the line orientation matching task, for several days of training without feedback. The data were analyzed with single-subject analyses of variance (ANOVAs), using the interaction term for days of training and training blocks per day as the error term. There were significant practice effects for all observers, as is shown by the significant linear trends [for P.P. and A.S., $F(1,44)>7.5$; for M.L., $F(1,77)=17.28$, all with $p<.01]$ and the significant differences between the first and the last day of training [all with $F(1,11)>13$, $p<.01]$. As in the results of the previous experiments, there was no evidence of learning within a daily session

(A)

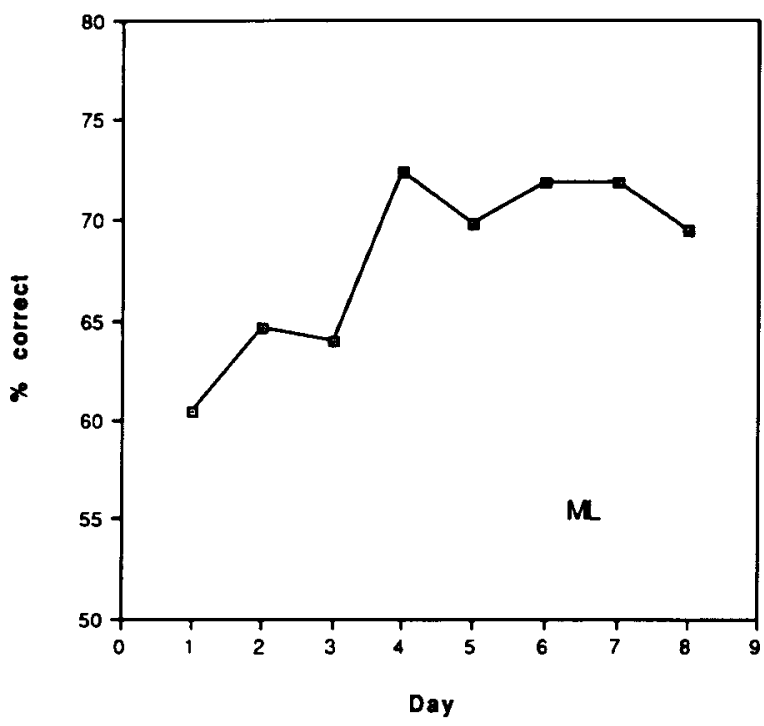

(B)

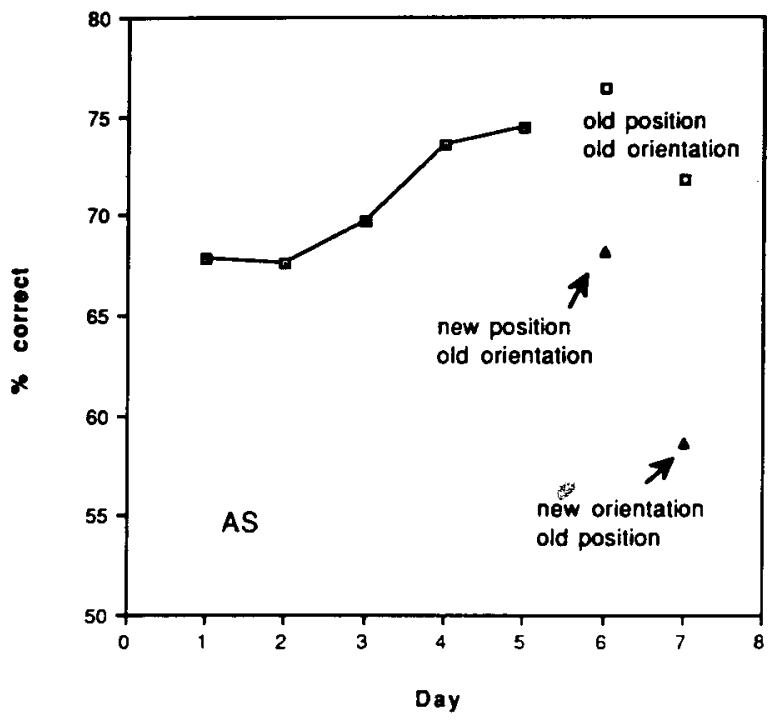

(C)

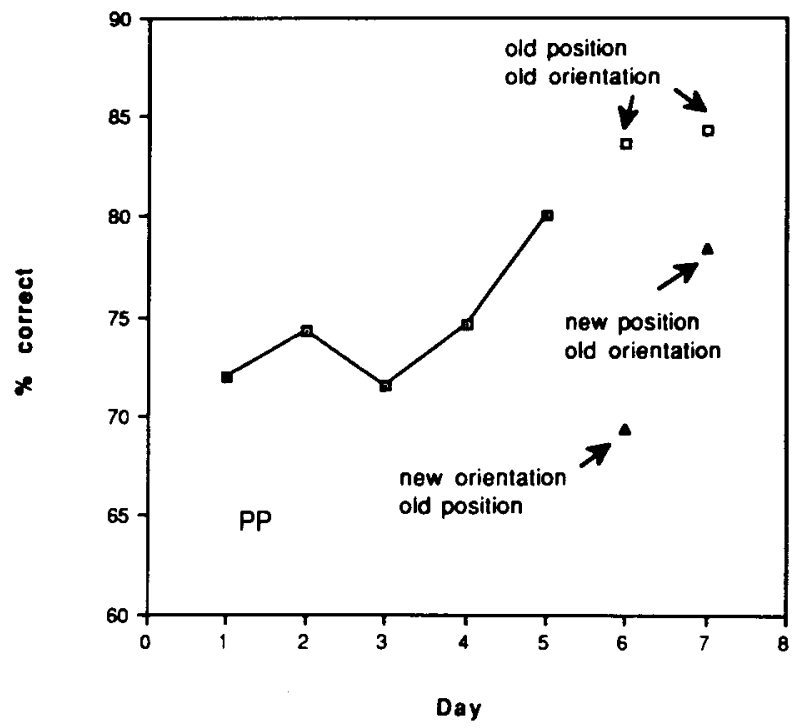

Figure 2. Results of three no-feedback observers with several days of training, with 12 blocks $\times 40$ trials each day (Experiment 2 ). 
$\left(F<1.5\right.$ for all observers) ${ }^{4}$ A.S. and P.P. were given 2 additional testing days, where the lines were either shown in an untrained corner or rotated $90^{\circ}$ in half of the blocks. For both observers, accuracy dropped substantially when the lines appeared in a new location. ${ }^{5}$ Furthermore, a change in stimulus orientation, even though the visual field to be attended to remained the same, seemed to create even more difficulty. This rules out the possibility that location-specific improvement is caused by improvement in focusing attention at the trained location (cf. Bennett \& Westheimer, 1991). If the trend toward an actual decrement in new orientations is real, this is intriguing and worthy of further investigation.

Karni and Sagi (1991) suggested that a few hours of "synaptic consolidation" after practice might be required in order to retain perceptual learning effects. But the absence of immediate improvement when subjects were trained without feedback can also be explained in terms of the buildup of fatigue. In fact, Karni and Sagi's subjects were required to do $800-1,000$ training trials per daily session, which would seem likely to have been quite fatiguing.

Although the evidence is not as decisive as one might wish, a consolidation hypothesis is not easily reconcilable with the results of Experiment 1, in which immediate improvement was observed for subjects given block feedback during training. Fahle, Poggio, and Edelman (1992) have also found immediate learning effects after subjects were trained with vernier discrimination for less than $1 \mathrm{~h}$. We therefore tentatively conclude that the fatigue explanation is more plausible, given our results.

\section{EXPERIMENT 3}

Perceptual learning without explicit feedback suggests that the visual system can calibrate its responses to environmental stimuli in an unsupervised manner. One wellcharacterized mechanism of this kind is adaptation to the mean of a background illumination (Barlow, 1989). In cats, the response range of ganglion cells shifts about 0.7 $\log$ unit as the intensity of a steady background light increases by every $1 \log$ unit, thus centering the response range around the mean background luminance (Walraven, Enroth-Cugell, Hood, MacLeod, \& Schnapf, 1990). Mere exposure to a background light is enough for adaptation. Similarly, Houck and Hoffman (1986) have shown that McCollough aftereffects can occur whether or not the adaptation stimuli are within a perceiver's focus of spatial attention.

On the other hand, recent studies (e.g., Treisman \& Gormican, 1988) suggest that discrimination of small differences in line orientation may require serial processing. In Experiment 3, we directed subjects to judge the brightness level of the line stimuli, but not their orientation. We then tested to see whether perceptual learning for line orientation discrimination can be produced by mere exposure to the oriented stimuli (over a relatively long time scale), when orientation is irrelevant to the training task.

\section{Method}

In the training phase of this experiment, 8 observers were asked to discriminate the relative brightness of the same lines as those used in the previous experiments. The observers were divided into two groups. One group was given an easy brightness discrimination task, in which each line was presented at a luminance of 48 or $21 \mathrm{~cd} / \mathrm{m}^{2}$. The other group was given a more difficult discrimination, in which each line was at a luminance of 48 or $31 \mathrm{~cd} / \mathrm{m}^{2}$. The observers were asked to decide whether two lines presented successively were the same in brightness or not. The lines also differed in orientation, and orientation was varied orthogonally to luminance level. This would ensure that subjects' judgments of relative brightness would not be based on the orientations of the lines.

Baseline measurements of orientation discrimination accuracy were taken on the first day of the experiment, with the lines appearing in one of the two (diagonal) corners in alternate blocks. The observers then practiced brightness discrimination with the lines presented in one particular corner only. Half of the subjects practiced with the upper-left corner and the other half with the bottomright corner. Within each group, half were given vertically extended lines and the other half were given horizontally extended lines. After training, orientation discrimination was again assessed (in the trained and untrained corners). When orientation discrimination was measured, the luminance levels of the lines were set at 33 and $38 \mathrm{~cd} / \mathrm{m}^{2}$ for the easy and difficult (brightness discrimination) groups, respectively. These values lie between the bright and dim lines for each group.

Changes in luminance would not be expected to affect which orientation channels respond to the stimuli. Sclar and Freeman (1982) demonstrated that orientation selectivity in the cat's striate cortex is invariant with stimulus contrast, for example. In a pilot experiment, we changed the luminance but retained the location and orientation of the lines in the testing phase. We found complete transfer of the practice effect across luminance levels: both from bright to dim and from dim to bright.

There was absolutely no feedback in this experiment. If attending to the lines as a whole, but not to their orientations in particular, was sufficient to produce the improvement reported above, the trained corner should have a selective advantage over the untrained corner after practice with brightness discrimination. In other words, the effect of training should interact with stimulus locations. (Note that the first baseline orientation session was bound to produce some overall improvement that was not particular to one location.)

\section{Results and Discussion}

The results for the brightness discrimination task are plotted in Figure 3. The two curves represent the easy and the difficult discrimination conditions. Each data point is the mean of the performance of four subjects. ${ }^{6}$ The data were analyzed with a 2 (easy, difficult) $\times 5$ (Days 1-5) mixed-design ANOVA. Judgment accuracy was higher in the easy condition throughout the course of training. The average percentages of correct response for the easy and the difficult conditions were $64.3 \%$ and $56.4 \%$. This difference is statistically significant $[F(1,6)=11.36$, $p<.05]$. The effect of days of training is also significant $[F(4,24)=4.31, p<.01 ; p<.05$ with Box adjustment of degrees of freedom, Hayes, 1988]. For example, accuracy increased from $58.8 \%$ to $69.0 \%$ after 5 days of training with the easy discrimination task. But 


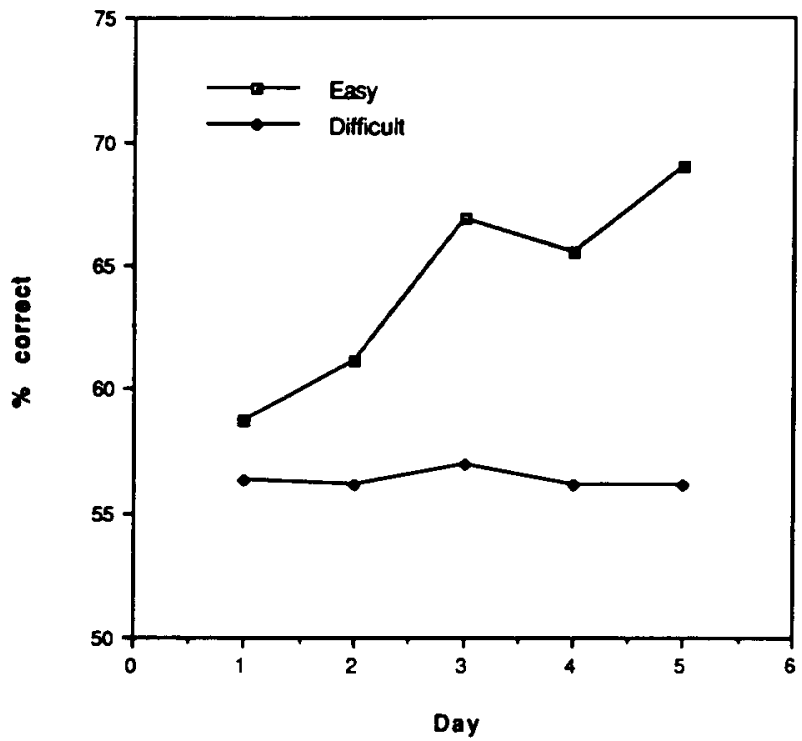

Figure 3. Percentages of correct brightness discrimination in the easy and difficult conditions across 5 days of training in Experiment 3.

improvement was found in this condition only. This is supported by a statistically significant interaction $[F(4,24)=4.08, p<.05 ; p<.05$ with Box adjustment]. Probably the difficult discrimination condition was too difficult for our subjects. Their performance was slightly above chance level, and it did not change much throughout the days of training.

Discrimination accuracies with the orientation task before and after the brightness discrimination training are shown in Figure 4. The results are plotted separately for the two groups of subjects, at the trained (where they practiced brightness judgment) and the untrained locations. The data were analyzed with a 2 (Groups 1-2) $\times 2$ (baseline, test phase) $\times 2$ (trained, untrained locations) ANOVA, with repeated measures on the last two factors. Although Group 1 scored better than Group 2 in the brightness task, it did not differ significantly in the orientation task $[F(1,6)=3.44, p>.05]$. This is not unexpected, because the two groups differed mainly with respect to the ease of the brightness discrimination task. Overall, orientation discrimination was slightly more accurate on the test phase $(73.3 \%)$, relative to the baseline condition $(68.4 \%)$. But this improvement was not significant $[F(1,6)=3.36, p>.05]$. Averaged over the two groups, the improvement in the trained corner was $6.5 \%$ (from $68.6 \%$ to $75.1 \%$ ) and the improvement in the untrained corner was $3.2 \%$ (from $68.3 \%$ to $71.5 \%$ ). But the advantage of the trained corner was far from significant $[F(1,6)=2.92, p>.05]$. This is true irrespective of groups - the three-way interaction of group, phase, and corner was not significant $[F(1,6)<1]$. In other words, although some subjects did not improve in the brightness discrimination task (probably because it was too difficult) while some did, this had no observable effect on the orientation discrimination task.

To examine the results further, we also carried out single-subject ANOVAs like those in Experiment 2, using all the interaction terms involving blocks of training as error terms, for each individual subject. The analyses showed that 2 subjects in the difficult and 1 subject in the easy brightness discrimination condition improved significantly $(p<.05)$ in the orientation task. But the improvement at the trained location was never much different from the improvement at the untrained location. This contrasts sharply with the results of the last experiment, in which orientation discrimination accuracies at the untrained location were much lower than those at the trained location (after training) for each subject.

In short, there was no evidence that repeated exposure to the lines enhances the ability to discriminate their orientations. Although our subjects did attend to the luminance level of the lines, the training with brightness discrimination did not help orientation discrimination.

\section{GENERAL DISCUSSION}

Our experiments demonstrated retinally local improvement in orientation discrimination in the absence of feedback. The improvement might be due to changes in the tuning of some orientation selective mechanisms, or to an increase in the efficiency of the connections between earlier mechanisms and the orientation channels. In any case, the results suggest that sensitivity to contour orientation can be modified by what are usually termed "unsupervised learning mechanisms" (Barlow, 1989; Rumelhart \& Zipser, 1985). Since improvement did not occur when orientation of the stimuli was irrelevant to

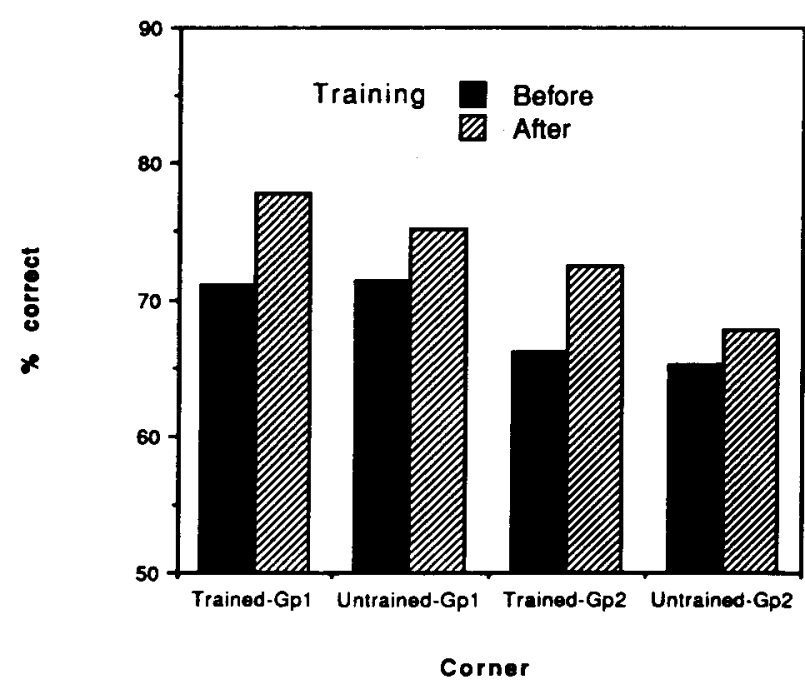

Figure 4. Percentages of correct orientation discrimination before and after practice with brightness judgment in Experiment 3. Trained-Gp1 = performance of the easy brightness discrimination group at the trained corner. 
the training task, the learning depends on the observer's having an appropriate cognitive set. Physiological evidence has shown that neural responses to visual features can sometimes be modulated by their behavioral significance (Haenny, Maunsell, \& Schiller, 1988). Our observations further demonstrate that learning effects in visual areas that are still retinotopically organized can be so modulated as well.

In a number of ways, our results parallel Karni and Sagi's (1991) investigation of orientation-based texture discrimination. But our perceptual task may tap a different level of processing. The learning effect that they found was specific to the location of the targets and to the orientation of the uniform background elements. It was not specific to the orientation of the discrepant texture patch. This implies that the visual system was getting more sensitive in detecting an orientation gradient at the texture border. Derivation of an orientation gradient presumably requires a second-level unit that receives input from some orientation-sensitive mechanisms at the lower level. The learning effect Karni and Sagi found seems likely to be occurring at the second level. On the other hand, our results suggest that the selectivity of the lower level mechanisms can be altered by perceptual experience as well. Despite this difference, the conditions in which perceptual learning occurs are strikingly similar across the two studies: learning does not require feedback, but the dimension to be learned has to be task relevant.

\section{REFERENCES}

Ball, K., \& SekUler, R. (1987). Direction-specific improvement in motion discrimination. Vision Research, 27, 953-965.

BARLOW, H. B. (1989). Unsupervised learning. Neural Computation, 1, 295-311.

Bennett, R. G., \& Westheimer, G. (1991). The effect of training on visual alignment discrimination and grating resolution. Perception \& Psychophysics, 49, 541-546.

Blakemore, C., \& Campbell, F. W. (1969). On the existence of neurons in the human visual system selectively sensitive to the orientation and size of retinal images. Journal of Physiology, 203, 237-260.

Blakemore, C., \& Cooper, G. F. (1970). Development of the brain depends on the visual environment. Nature, 228, 477-478.

Fahle, M., Poggio, T., \& Edelman, S. (1992, May). Generalization of learning in vernier acuity. Paper presented at the meeting of the Association for Research in Vision and Ophthalmology, Sarasota, FL.

FIORENTINI, A., \& BERARDI, N. (1981). Learning in grating waveform discrimination: Specificity for orientation and spatial frequency. Vision Research, 21, 1149-1158.

GARNER, W. R. (1974). The processing of information and structure. Potomac, MD: Erlbaum.

GreEN, D. M., \& SwETS, J. A. (1966). Signal detection theory and psychophysics. New York: Wiley.

HaenNy, P. H., MAunsell, J. H. R., \& Schiller, P. H. (1988). State dependent activity in monkey visual cortex. Experimental Brain Research, 69, 245-259.

HAYES, W. L. (1988). Statistics. Fort Worth, TX: Holt, Rinehart \& Winston.

Houck, M. R., Hofrman, J. E. (1986). Conjunction of color and form without attention: Evidence from an orientation-contingent color aftereffect. Journal of Experimental Psychology: Human Perception \& Performance, 12, 186-199.

HubEL, D. H., WIESEL, T. N. (1962). Receptive fields, binocular interaction and functional architecture in the cat's visual cortex. Journal of Physiology, 160, 106-154.
KARNI, A., \& SAGI, D. (1991). Where practice makes perfect in tex ture discrimination: Evidence for primary visual cortex plasticity. Proceedings of the National Academy of Sciences, 88, 4966-4970.

Kimble, G., Shatel, R. B. (1952). The relationship between two kinds of inhibition and the amount of practice. Joumal of Experimental Psychology, 38, 355-359.

MCKeE, S. P., \& Westheimer, G. (1978). Improvement in vernier acuity with practice. Perception \& Psychophysics, 24, 258-262.

RumelharT, D. E., \& Zipser, D. (1985). Feature discovery by competitive learning. Cognitive Science, 9, 75-112.

Sclar, G., \& FreEman, R. D. (1982). Orientation selectivity in the cat's striate cortex is invariant with stimulus contrast. Experimental Brain Research, 46, 457-461.

Treisman, A. (1986). Properties, parts, and objects. In K. R. Boff, L. Kaufman, \& J. P. Thomas (Eds.), Handbook of perception and human performance (Vol. 2, pp. 35.1-35.70). New York: Wiley.

Treisman, A., \& Gelade, G. (1980). A feature-integration theory of attention. Cognitive Psychology, 12, 97-136.

Treisman, A., \& Gormican, S. (1988). Feature analysis in early vision: Evidence from search asymmetries. Psychological Review, 95, $15-48$.

Van Essen, D. C., Maunsell, J. H. R. (1983). Hierarchical organization and functional streams in the visual cortex. Trends in Neuroscience, 6, 370-375.

Vogels, R., \& Orban, G. A. (1985). The effect of practice on the oblique effect in line orientation judgments. Vision Research, 25, 1679-1687.

Walraven, J., Enroth-Cugell, C., Hood, D. C., Macleod, D. I. A., \& SCHNAPF, J. L. (1990). The control of visual sensitivity. In L. Spillman \& J. S. Werner (Eds.), Visual perception (pp. 53-101). San Diego, CA: Academic Press.

WINER, B. J. (1962). Statistical principles in experimental design. New York: McGraw-Hill.

WOODWORTH, R. S., \& SCHLOSBERG, H. (1955). Experimental psychology. New York: Holt.

\section{NOTES}

1. The improvement has to be indicative of increased sensitivity instead of better decision criterion, because $d^{\prime}$, a criterion-free sensitivity measure, shows the same pattern of improvement. (See Green \& Swets, 1966.)

2. The same conclusion was reached when Blocks 1, 2, and 3; Blocks 7,8 , and 9; and Blocks 10,11 , and 12 were analyzed, except that the difference between Blocks 7, 8, and 9 and Blocks 10,11 , and 12 in the no-feedback condition was significant $[F(1,15)=10.15, p<.01]$, indicating that accuracies for the transfer blocks were significantly worse than accuracy at the end of training. The rate of improvement after transfer was not significantly faster than that at initial learning.

3. P.P. was not given $I$ and 1 .

4. We assumed that the effects of days and blocks of training were additive. This assumption might have inflated the $M S_{\text {residual in case there }}$ was a real day $\times$ block interaction (see Winer, 1962, p. 216). Anyway, the $M S$ for blocks of training was small for all three subjects.

5. For A.S., accuracy was $8.5 \%$ higher than on the first day of training (the odd-numbered blocks) when the lines appeared at the trained location on the test day; and accuracy was only $1.5 \%$ higher than on the first day of training (the even-numbered blocks) when the lines appeared at the untrained location. The figures for Observer P.P. are $15.2 \%$ and $3.5 \%$.

6. Owing to experimenter error, 1 subject in the difficult discrimination condition was given 4 days of training (instead of 5). In order to do the ANOVA, her results from Day 4 were duplicated for Day 5. This adjustment, however, should not affect our interpretations of the results. Two subjects in the easy condition and 2 in the difficult condition made more than 5 errors with the central alphanumeric characters on one of the training days. On the average, they made 2.2 errors per day.

(Manuscript received November 7, 1991; revision accepted for publication May 13, 1992.) 